\title{
German health interview and examination survey for adults (DEGS) - design, objectives and implementation of the first data collection wave
}

Christa Scheidt-Nave*, Panagiotis Kamtsiuris, Antje Gößwald, Heike Hölling, Michael Lange, Markus A Busch, Stefan Dahm, Rüdiger Dölle, Ute Ellert, Judith Fuchs, Ulfert Hapke, Christin Heidemann, Hildtraud Knopf, Detlef Laussmann, Gert BM Mensink, Hannelore Neuhauser, Almut Richter, Anke-Christine Sass, Angelika Schaffrath Rosario, Heribert Stolzenberg, Michael Thamm and Bärbel-Maria Kurth

\begin{abstract}
Background: The German Health Interview and Examination Survey for Adults (DEGS) is part of the recently established national health monitoring conducted by the Robert Koch Institute. DEGS combines a nationally representative periodic health survey and a longitudinal study based on follow-up of survey participants. Funding is provided by the German Ministry of Health and supplemented for specific research topics from other sources.

Methods/design: The first DEGS wave of data collection (DEGS1) extended from November 2008 to December 2011. Overall, 8152 men and women participated. Of these, 3959 persons already participated in the German National Health Interview and Examination Survey 1998 (GNHIES98) at which time they were 18-79 years of age. Another 4193 persons 18-79 years of age were recruited for DEGS1 in 2008-2011 based on two-stage stratified random sampling from local population registries. Health data and context variables were collected using standardized computer assisted personal interviews, self-administered questionnaires, and standardized measurements and tests. In order to keep survey results representative for the population aged 18-79 years, results will be weighted by survey-specific weighting factors considering sampling and drop-out probabilities as well as deviations between the design-weighted net sample and German population statistics 2010.

Discussion: DEGS aims to establish a nationally representative data base on health of adults in Germany. This health data platform will be used for continuous health reporting and health care research. The results will help to support health policy planning and evaluation. Repeated cross-sectional surveys will permit analyses of time trends in morbidity, functional capacity levels, disability, and health risks and resources. Follow-up of study participants will provide the opportunity to study trajectories of health and disability. A special focus lies on chronic diseases including asthma, allergies, cardiovascular conditions, diabetes mellitus, and musculoskeletal diseases. Other core topics include vaccine-preventable diseases and immunization status, nutritional deficiencies, health in older age, and the association between health-related behavior and mental health.
\end{abstract}

Keywords: Health, Indicators, Survey, Examination, Adults, Germany, Population, Trends, Follow-up

\footnotetext{
* Correspondence: Scheidt-NaveC@rki.de

Department of Epidemiology and Health Monitoring, Robert Koch Institute, General-Pape-Strasse 62-66, 12101 Berlin, Germany
} 


\section{Background}

In Germany, several health interview and examination surveys have been carried out since 1984. These surveys addressed different subsets of the population and were conducted at irregular intervals. In the framework of the German Cardiovascular Prevention Study (GCP), residents of former West Germany 25-69 years of age were surveyed in three independent consecutive surveys conducted in 1984-86 $(\mathrm{n}=4790), 1987-89(\mathrm{n}=5335)$, and 1990-91 $(\mathrm{n}=5311)$. The surveys were restricted to the population in private households and to those who could sufficiently speak German [1]. In 1991-92, the Survey East was conducted, in order to provide comparable health data for adults 18-79 years residing in private households of former East Germany. Data of the Survey East and the 1990-91 GCP survey were pooled for analyses of health data in the reunified Germany. Nearly a decade later, the German National Health Interview and Examination Survey 1998 (GNHIES98) conducted under the auspices of the Robert Koch Institute (RKI) was the first nationally representative health survey of non-institutionalized adults 18-79 years in reunified Germany [2]. It opened the perspective for analyzing regional differences in health trends in comparison to the 1990-92 data [3]. In 2003-2006, the RKI conducted the German Health Interview and Examination Survey for Children and Adolescents (KiGGS). Including a total of 17641 study participants, KiGGS established a large nationally representative health database for the non-institutionalized German population 017 years of age [4].

In 2008, the German Ministry of Health commissioned the RKI to implement a system of health studies for continuous health monitoring of the non-institutionalized population [5]. Survey components include: (1) annual health interview surveys (German Health Update, GEDA) conducted in large representative and independently drawn samples of the population 18 years and older, (2) a national cohort study of children and adolescents in Germany based on continuous follow-up of the KiGGS study population, (3) periodically repeated interview and health examination surveys of the population 18-79 years of age (German Health Interview and Examination Survey for Adults, DEGS). We here describe the design, objectives and implementation of the first wave of data collection in DEGS (DEGS1), which was carried out from November 2008 to November 2011.

\section{Methods/design}

\section{Study design}

DEGS is primarily designed as a periodically repeated national health interview and examination survey of adults in Germany. The target population comprises adults 18-79 years of age with permanent residence in Germany according to local population registries. In order to lay the ground for longitudinal studies, persons who had participated in the 1998 national health interview and examination survey (GNHIES98) were invited to take part in DEGS1, provided they had agreed to be recontacted and were still contactable [6]. Representativeness of DEGS1 as a cross-sectional survey is maintained by extending the population sample based on a two-stage sampling procedure according to power analyses and sample size estimations as described below.

The implementation of DEGS1 conforms to the principles outlined in the Declaration of Helsinki [7] and to the German Federal Data Protection Act. The DEGS1 study protocol was consented with the Federal and State Commissioners for Data Protection and approved by the Charité-Universitätsmedizin Berlin ethics committee in September 2008 (No. EA2/047/08). Participants provided written informed consent prior to the interview and examination.

\section{Study population}

Overall 17410 persons, of whom $\mathrm{N}=6402$ or $37 \%$ were GNHIES98 participants, were invited to participate in DEGS1. After excluding non-deliverable survey contacts, persons unable to provide written informed consent and persons with insufficient German language skills, the overall net sample consisted of 16305 adults, of whom $\mathrm{N}=6358$ or $39 \%$ were GNHIES98 participants. Overall, 8152 adults (4283 women; 3869 men) participated in DEGS1. Total numbers of the study population and response rates are summarized by age group and GNHIES98 participation status in Table 1. Response rates were consistently higher among GNHIES98 participants compared to newly sampled individuals.

Both interview and examination data are available for $89 \%$ of the study population $(\mathrm{N}=7238 ; 3765$ women,

Table 1 Study population and response rates in DEGS1

\begin{tabular}{|c|c|c|c|c|c|}
\hline \multirow[b]{2}{*}{ Age group (yrs) } & \multirow{2}{*}{$\frac{\text { Total }}{\mathrm{N}}$} & \multicolumn{2}{|c|}{$\begin{array}{l}\text { GNHIES98 } \\
\text { participants }\end{array}$} & \multicolumn{2}{|c|}{$\begin{array}{l}\text { Newly sampled } \\
\text { participants }\end{array}$} \\
\hline & & $\mathrm{N}$ & Response & $N$ & Response \\
\hline$\overline{18-29}$ & 1073 & $19^{\mathrm{a}}$ & & 1054 & $40 \%$ \\
\hline $30-39$ & 1014 & 427 & $49 \%$ & 587 & $40 \%$ \\
\hline $40-49$ & 1539 & 901 & $63 \%$ & 638 & $44 \%$ \\
\hline $50-59$ & 1592 & 928 & $69 \%$ & 664 & $46 \%$ \\
\hline $60-69$ & 1537 & 867 & $73 \%$ & 670 & $47 \%$ \\
\hline $70-79$ & 1233 & 653 & $65 \%$ & 580 & $38 \%$ \\
\hline 18-79 & 7988 & 3795 & $64 \%$ & 4193 & $42 \%$ \\
\hline $80-91$ & 164 & 164 & $38 \%$ & & \\
\hline$\overline{18-91^{a}}$ & 8152 & 3959 & $62 \%$ & 4193 & $42 \%$ \\
\hline
\end{tabular}

${ }^{\mathrm{a}} \mathrm{GNHIES} 98$ participants were $28-91$ years of age at the time of DEGS1. 
3473 men). The remainder of the study population $(\mathrm{N}=914 ; 518$ women, $396 \mathrm{men})$ have interview data only, because they were unable or unwilling to come to one of the local study centers. Cross-sectional and trend analyses will be restricted to 7988 DEGS1 participants 18 to 79 years of age, with examination as well as interview data available for 7116 of these. Longitudinal studies will include a total of 3959 persons 28-91 years of age who participated in DEGS1 as well as in the GNHIES98.

\section{Sampling procedure}

The DEGS1 sampling protocol was developed in cooperation with the Leibniz Institute for the Social Sciences (GESIS), Mannheim, Germany [8]. Like previous German health interview and examination surveys $[1,2,4]$ DEGS1 is based on two-stage stratified cluster sampling. Primary sampling units (PSUs) are the communities. PSUs were sampled from a list of German communities stratified according to districts and the BIK classification system, which takes into account the grade of urbanization, regional population density, and administrative borders [9]. Sampling was done with probability proportional to community size using the Cox procedure for controlled rounding [10]. Selected metropolitan areas were generally represented by several PSUs. Selected communities with less than 1000 inhabitants were combined with neighboring small communities to form a single PSU. Within PSUs, random samples of individuals, stratified by 10 -year age groups, were drawn from local population registers.

Since DEGS1 included GNHIES98 participants, the DEGS1 PSU sample had to build upon a total of 120 PSUs previously sampled for the 1998 survey. In order to remain representative at the population level, additional PSUs $(\mathrm{N}=60)$ were sampled. In newly added PSUs, all participants were newly sampled. Within GNHIES98 PSUs, only newly recruited individuals are included in the age group 18-28 years due to the aging of the GNHIES98 cohort by $10-14$ years. Persons 30 years and older were sampled as necessary, in order to replace the number of participants expected to decline participation in DEGS1 or to be lost to follow-up. The number of newly sampled PSUs and individuals per PSU was determined by statistical power and sample size considerations as described below.

\section{Statistical power and sample size calculations}

Given the cluster design, simple variance estimates of prevalence estimates will underestimate total variability and hence estimation error, because individuals within PSUs can be assumed to show greater similarities than individuals randomly selected from the entire population of Germany. The increase in estimation error is quantified by the design effect (Deff). Total sample size needs to be increased in proportion to Deff to attain the same level of precision as in simple random sampling [11]. Deff depends on the average number of individuals within PSUs $(m)$ and the degree of similarity within clusters which is estimated by the intra class correlation coefficient (ICC): Deff $=1+I C C(m-1)$ [12]. It is therefore more efficient to increase the total number of PSUs rather than $m$, in order to keep the total sample size as small as possible. Reasonably precise estimation of a sex and 10-year-age group-specific prevalence of 1\% (95\% $\mathrm{CI}$ to be attained: $0.5-2.1 \%$ ) would require a total sample size of about 7500. In this calculation, Deff is ignored as the number of persons in a PSU within any sex-agegroup can be expected to be very small. Analyses of GNHIES98 data showed that ICC can be assumed to take on values between 0.005 and 0.036 . Based on the experience that survey logistics permit the examination of 40-50 persons per PSU, design effects would be $4-15 \%$ higher with 50 instead of 40 persons seen per PSU. The required precision could most efficiently be reached based on a total number of 180 PSUs and an average of $m=42$ persons seen per PSU, yielding a total sample size of $180 \times 42=7560$ [8]. This sample size also proved to provide $90 \%$ power to detect a $3 \%$ change in overall obesity prevalence in analyses for time trends at the $5 \%$ significance level based on twosided tests [13].

\section{Recruitment of the study population}

Eligible persons were invited to participate in the survey by letter. An information booklet illustrated the study objectives, procedures and logistics. A response card was enclosed to fill in telephone numbers and preferred contact times. Participants were offered a small monetary incentive.

Invitation letters were sent approximately five weeks prior to the survey visit. In order to optimize response rates, survey activities were announced in local newspapers, and if possible, on local television and radio stations. In addition, small survey reports were published at regular intervals in national medical and public health journals. Persons willing to participate were contacted by telephone for scheduling and further information. A confirmation letter including the time of the appointment, location plan, and preparatory instructions was sent thereafter. Persons with appointments in the morning were asked to keep an overnight fast and not eat or drink anything but water before blood testing, unless they were diabetic. Persons with afternoon appointments were asked to fast for at least four hours, unless they were diabetic. Participants were asked to bring along immunization records as well as the original containers 
of all medications (prescription and over-the-counter) used within 7 days prior to the survey appointment.

Personal contact was sought to persons who had not responded to the invitation letter within four weeks. Exclusion criteria (such as insufficient language proficiency or inability to provide informed consent) were recorded. Persons willing to participate but facing organizational problems (e.g. lack of transportation, time constraints) were offered transportation and flexible scheduling, such as appointments early in the morning or in the evening.

\section{Field logistics}

Fieldwork in DEGS1 extended over three years. Data collection started on November 25, 2008 and ended on November 26, 2011. PSUs were successively visited by two mobile study teams according to a random touring schedule, in order to avoid a systematic bias of study results by seasonal or time trends. Duration of stay at a particular PSU was limited to one week. In order to include a sufficiently high number of study participants per PSU, eligible persons had to be scheduled for appointments ranging from 7:30 a.m. to 6:00 p.m. during the week and from 7:30 a.m. to 10:30 a.m. on Saturdays.

Study teams consisted of specifically trained health professionals, including a study physician and three technicians. Interviews and examinations took place in community-owned facilities that were rented for the purpose of the survey.

\section{Data collection procedures}

Data collection in DEGS1 was based on: (1) a semiquantitative self-administered food frequency questionnaire (FFQ); (2) automated assessment of currently used medications (AmEDa); (3) collection of urine and blood samples; (4) standardized measurements and physical performance tests $(\mathrm{M} / \mathrm{T})$; (5) a standardized physicianadministered computer-assisted personal interview (CAPI); (6) a standardized self-filled questionnaire (SFQ).

The FFQ questionnaire was mailed to study respondents along with the confirmation letter. It included instructions and examples for completion. Participants were asked to take the completed questionnaire to the survey appointment.

AmEDa as well as standardized measurements and tests were conducted by study technicians. Unique product identifiers (Pharmazentralnummer, PZN) on original drug containers brought to the survey site were scanned and automatically coded according to the latest version of the WHO Anatomical Therapeutic Chemical (ATC) Classification System [14]. Information on medication source (doctor's prescription, over-the-counter, family medicine cabinet), indication, dose, frequency and duration of use, and intake within the past 24 hours was documented. Missing information due to incomplete matching (about $27 \%$ of scanned drugs) or missing original containers (about $1.5 \%$ of participants) was completed by telephone follow-up contacts and hand searching.

Body weight and body height were measured in underwear with shoes removed. Waist and hip circumferences were measured according to a standardized protocol. Three consecutive automated blood pressure measurements were taken on the right arm three minutes apart with participants sitting and having been at rest for five minutes.

Spot urine samples were collected and dipstick tested on site. Venous blood samples were collected using Vacutainer EDTA and gel tubes. The time of blood draw and number of hours since last meal were recorded. Participants who came in fasting were served a breakfast or snack thereafter. Blood was centrifuged and separated. Serum specimens as well as urine specimens were aliquoted and stored at $-40^{\circ} \mathrm{C}$ within one hour. EDTA whole blood tubes were shaken and kept in the original collection tubes at $4^{\circ} \mathrm{C}$. A $100 \mu \mathrm{l}$ whole blood aliquot intended for folate analysis was hemolyzed under light protection and stored at $-40^{\circ} \mathrm{C}$. A full blood count was performed on site. Additional whole blood, serum and urine specimens were transported by car at $-40^{\circ} \mathrm{C}$ at the end of each survey week for further analysis and storage at the central epidemiology laboratory unit at the Robert Koch Institute, Berlin. Clinical chemistry and allergic sensitization analyses were performed within 6-8 weeks. Specific analyses and confirmatory tests relating to the diagnosis of infectious diseases were commissioned to reference laboratories. Extra serum, urine and whole blood aliquots were stored at $-40^{\circ} \mathrm{C}$.

Applying the CAPI, study physicians obtained a detailed medical history including family history. The interview was administered in German language, hence participants had to be sufficiently proficient in speaking and understanding German. Self-administered questionnaires filled in at the survey site were available in different languages (German, Turkish, Serbo-Croatian, Russian, English). Foreign language versions were offered to participants with German as a foreign language who were able to communicate in German, but had limited capacities for reading and writing in German. Different versions of the questionnaire were used for the population of working age (up to 64 years of age) and for persons 65 years and above. A short version of the self-administered questionnaire restricted to core questions was offered to those who lacked the strength or time to answer the full-length questionnaire.

Completing the entire survey program required an average of three hours. GNHIES98 participants who had moved outside their PSU and were unable to come to 
the survey site were asked to complete a computerassisted telephone interview (CATI) covering CAPI questions and to answer a self-administered postal questionnaire.

\section{Objectives}

DEGS1 aims to analyze health status, health risks and resources, functional capacity levels, and disability in the German adult population. Prevalence estimates will be obtained and time trends will be analyzed in comparison with data from the GNHIES98. Changes in health status and risk factors over time at the individual level will be studied among DEGS1 participants who already took part in the GNHIES98. Major study objectives include:

(1) To estimate the prevalence of diseases and risk factors with high public health impact and to identify differences according to socio-demographic characteristics, region of residence as well as changes over time;

(2) To analyze data on medication use and health care services utilization with regard to aspects of treatment effectiveness and quality of care;

(3) To investigate the association between mental health, physical health, and health-related behavior;

(4) To study patterns and determinants of co- and multimorbidity in the population 65 years and older in association with functional capacity levels, disability and health-related quality of life;

(5) To analyze allergic sensitization patterns and associations with manifest allergic disease in the population;

(6) To assess nutritional health risks and states of nutritional deficiency based on semiquantitative assessment of dietary habits and serum concentrations of various micronutrients (25-hydroxyvitamin D, vitamin B 12, iron, and ferritin in serum; serum and erythrocyte folate; urinary sodium excretion);

(7) To estimate iodine intake at the population level based on urinary iodine excretion;

(8) To examine individual changes in health status and cardiometabolic risk factors (e. g. body mass index; glycosylated hemoglobin A1c, serum lipids) over time.

\section{Constructs and instruments}

As summarized in Table 2, constructs and instruments applied in DEGS1 were kept compatible to those used in the GNHIES98 as far as possible. The CAPI covered a total of 32 health conditions including hepatitis (A, B, C, E), prevalent chronic diseases and cardiovascular events (stroke, myocardial infarction). Participants were asked whether a particular health condition was ever diagnosed by a physician, and if so, at what age or in what year the diagnosis was first made, and whether the condition required any current medical treatment or medication use. Persons with a lifetime history of a particular disease were asked, whether the disease was still present during the 12 months preceding the interview. Disease specific information was also obtained.

In addition to self-reported morbidity, detailed information on current medication surveyed by AmEDa, and various objective health measures will contribute to analyze health status and health risks in the adult population. Infectious diseases and immunization status will be evaluated based on self-reported childhood infections and vaccinations, immunization records, and laboratory test results. These include serum concentrations of antibody titers against several viruses and bacteria as well as nucleic acid amplification testing for confirmatory diagnosis of hepatitis $B$ and $C$ in serum and diagnosis of gonorrhea and chlamydia infections in urine. Medication use and objective health measures will help to validate self-reported chronic health conditions, to identify previously unknown disease and hidden health risks, and to analyze patterns of co- and multimorbidity as well as aspects of treatment effectiveness and quality of care. A list of chronic health conditions covered in DEGS1 and information available from interview and examination is given in Table 3.

Information on other facets of health, such as somatic symptoms and complaints, various aspects of mental health, and subjective health was collected via selfadministered questionnaires based on standardized instruments (Table 2). Self-administered questionnaires also served to collect detailed information on reproductive history among women, a history of benign prostate disease among men, injuries, and among persons 65 years and older, a history of fractures and falls as well as fear of falls.

Functional capacities were assessed by standardized questionnaires (vision and hearing impairment) and tests. Aerobic endurance fitness tests using submaximal endurance bicycle ergometry were restricted to the study population 18-64 years. Exclusion criteria were defined using the translated version of the Physical Activity Readiness Questionnaire (PARQ) [15]. Among persons 65 years of age and older, functional capacities relevant to daily life activities were assessed (Table 2). Isometric handgrip strength in both hands was measured while participants were standing upright using the Smedley S dynamometer. A simple test sequence was used to assess balance (Romberg test, semi-tandem, tandem, one-leg stand), lower leg muscle strength and coordination (fivechair rise test), and mobility (Timed "Up \& Go"). Cognitive functioning was assessed using the digit symbol substitution test (DSST) from the authorized German version of 


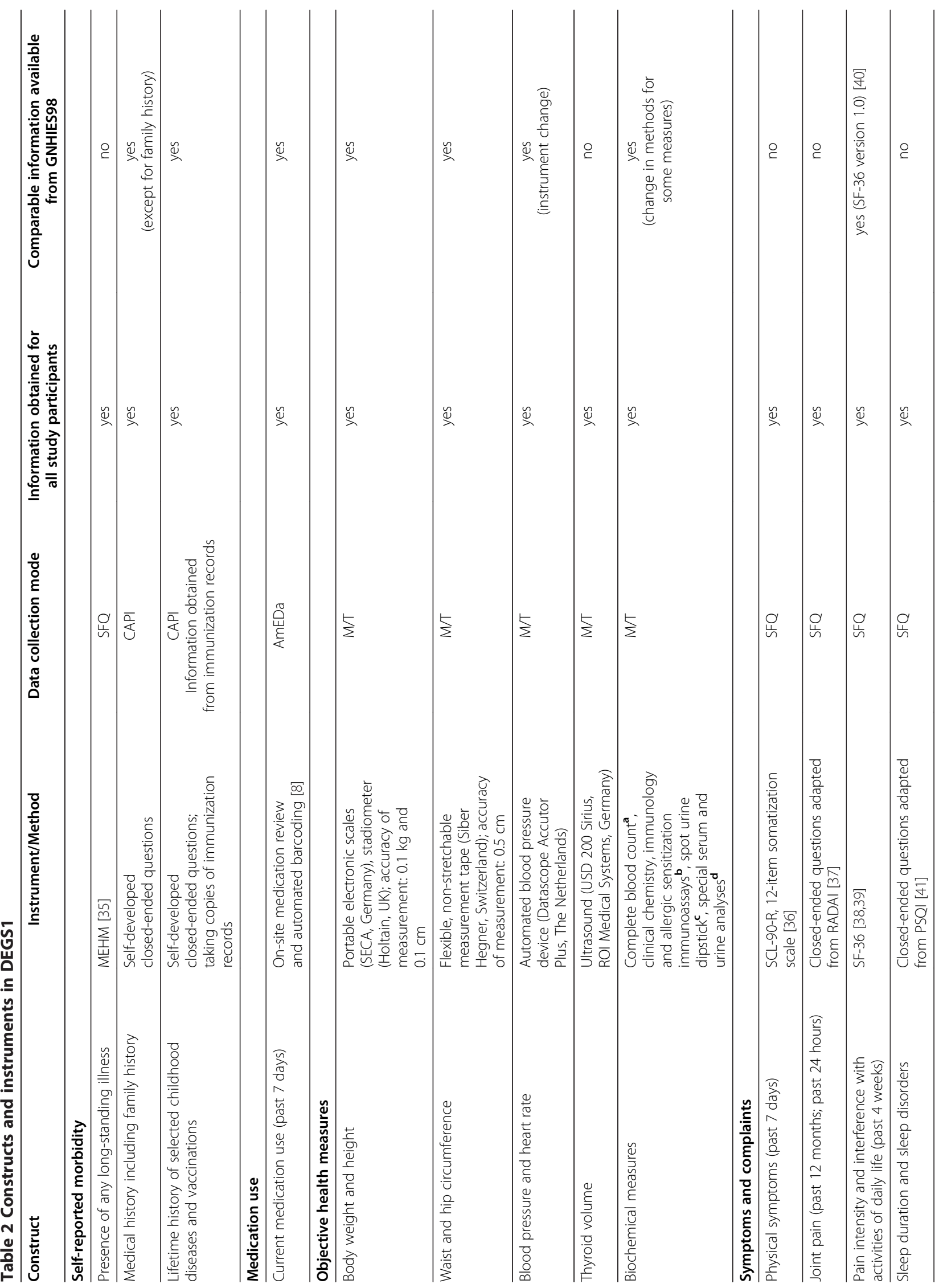




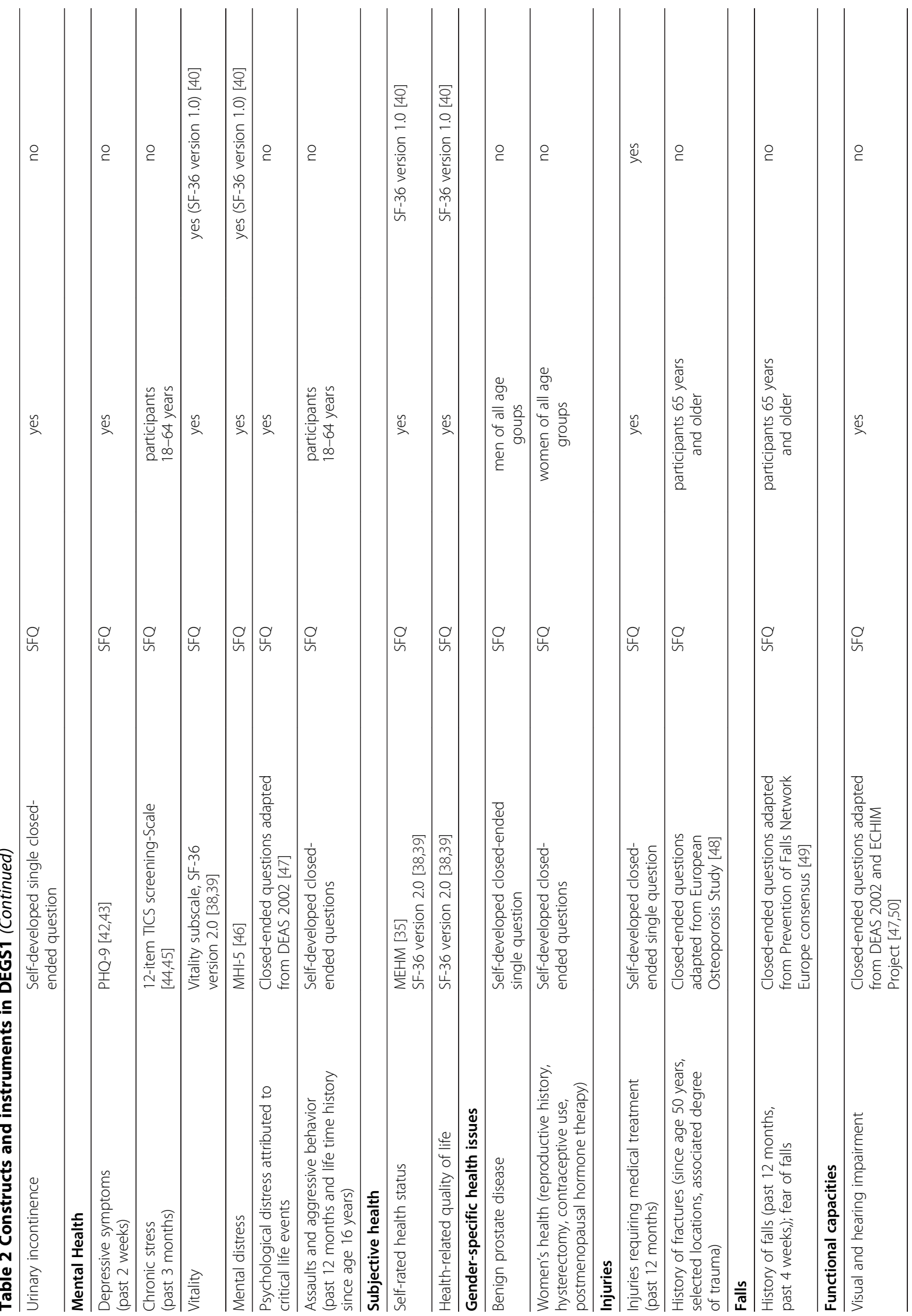




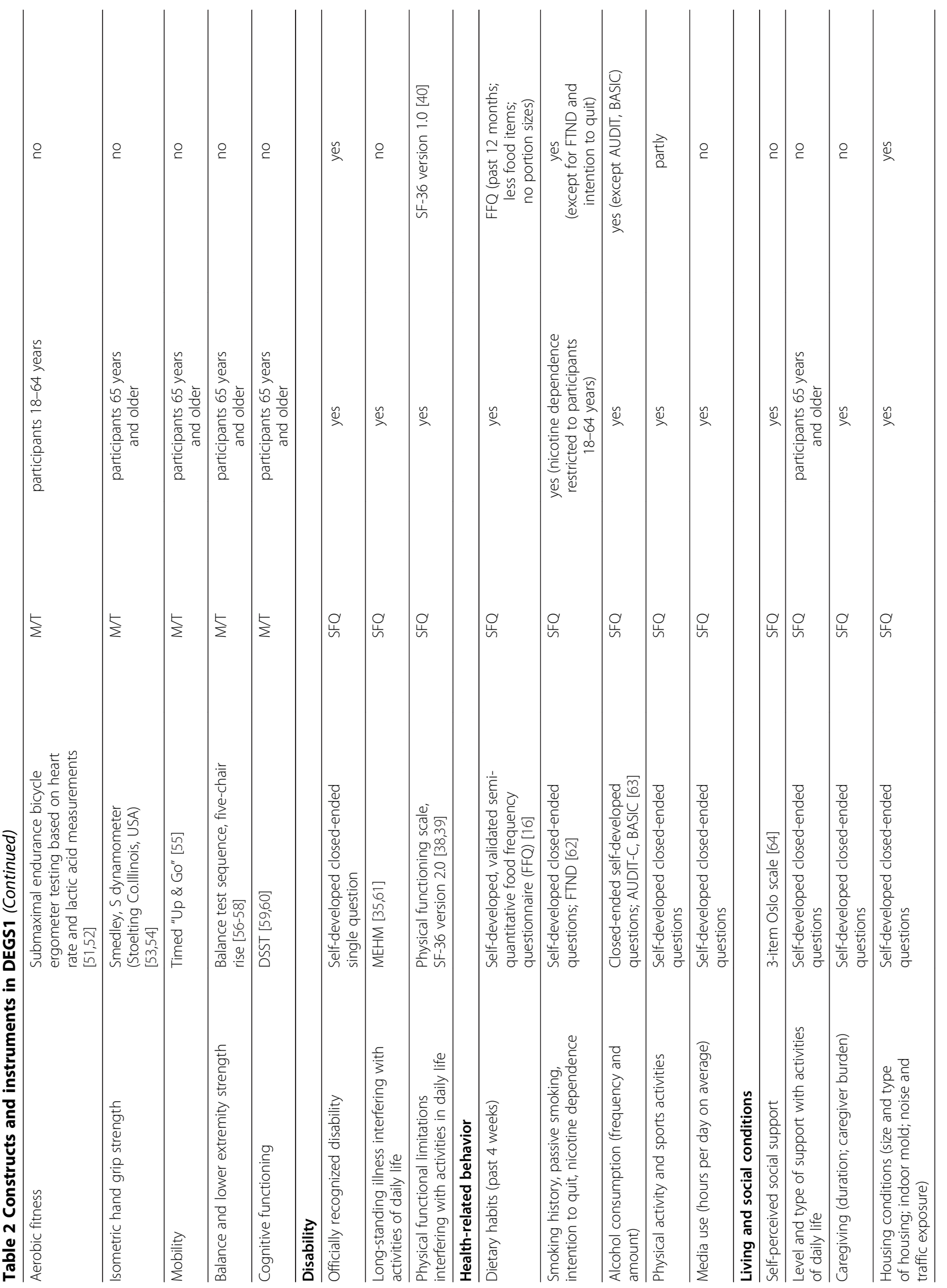




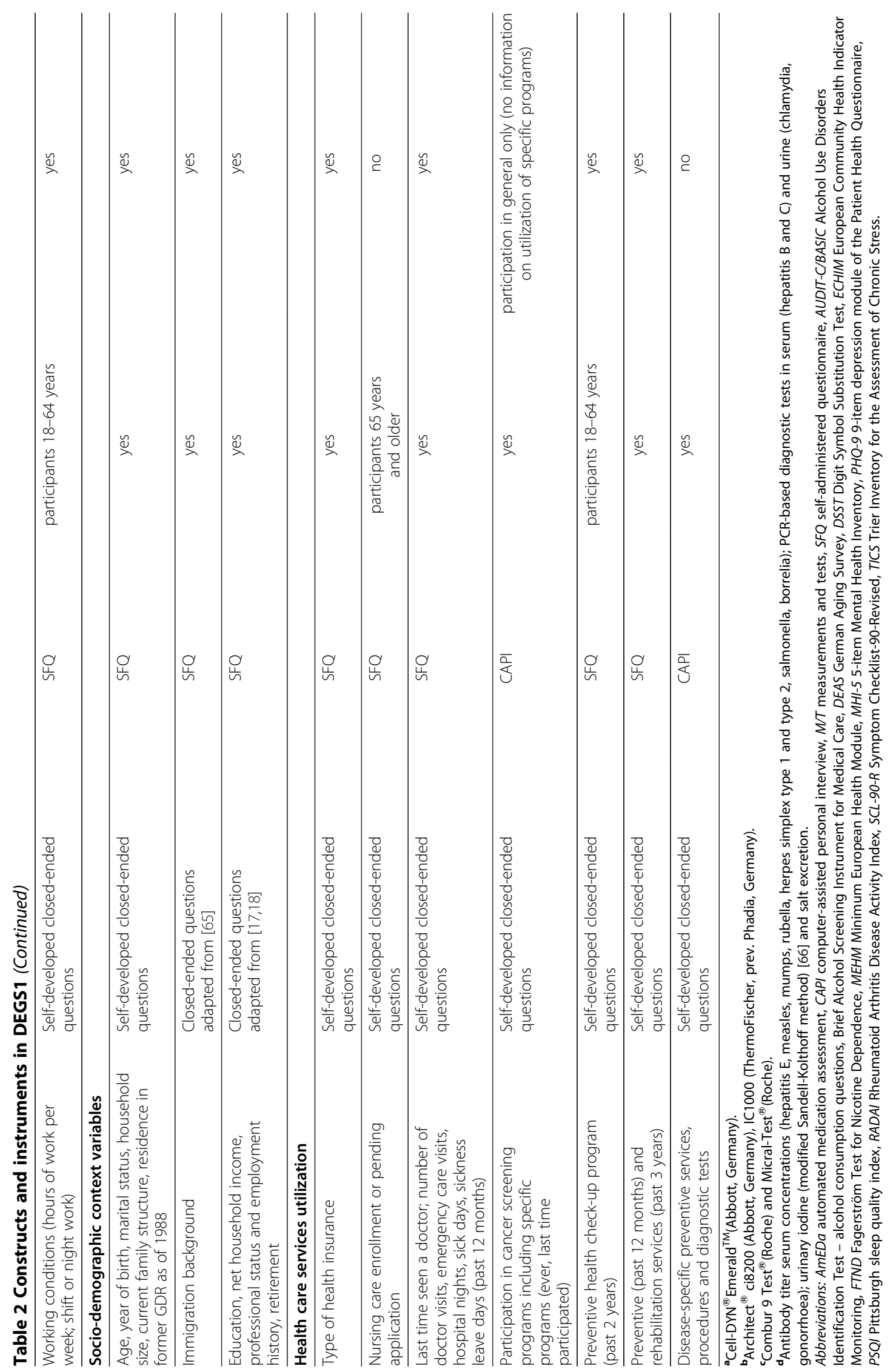


Table 3 Chronic health conditions and information available from health interview ${ }^{\mathrm{a}}$ and examination $^{\mathrm{b}}$ in DEGS1 Health condition Information

\section{Allergic disease}

Allergic contact eczema; allergic rhinitis; food allergy; atopic eczema; insect venom allergy; urticarial; other

Medical history and family history; current treatment;

lifetime history of allergy testing and specific immunotherapy Inhalation allergy screening tests, total serum IgE, allergen-specific IgE antibody tests against 52 specific allergens (food, pollen, contact, insect, inner room allergens)

\section{Cardiovascular disease}

Angina pectoris or other coronary heart disease

Medical history, current treatment; angina episodes and related hospitalizations, emergency room treatments, doctor visits during past 12 months; lifetime history of specific tests and surgical procedures

Myocardial infarction (MI)

Medical history and family history; current treatment; number of events; time of first and last Ml; lifetime history of specific tests and surgical procedures

Heart failure

Medical history; current treatment; acute exacerbations and related hospitalizations, emergency room treatments, doctor visits within past 12 months

Peripheral artery disease Medical history; current treatment

Stroke

Medical history and family history; current treatment; number of events, time of first and last event

Cardiometabolic conditions

Diabetes mellitus

Medical history including type of diabetes and family history; diagnosis during pregnancy; current treatment; treatment following diagnosis; presence of long-term diabetes complications; history of acute hyperor hypoglycemia and related hospitalizations, emergency room treatments, doctor visits within past 12 months; self-monitoring of blood glucose; time of last follow-up care (foot inspection, HbA1c assessment, eye background exam)

$\mathrm{HbA1c}$; serum concentrations of glucose, insulin, C-peptide, glycosuria ketonuria, microalbuminuria

Hypertension Medical history and family history; current treatment; self-monitoring; last time of blood pressure measurement by health professional; last self-measurement; history of hypertensive emergency and related hospitalizations, emergency room treatments, doctor visits during past 12 months

Blood pressure measurements

Dyslipidemia

Medical history; current treatment; serum total cholesterol, LDL- and HDL- cholesterol, triglycerides

Gout and hyperuricemia Medical history; current treatment; serum uric acid

\section{Gastrointestinal disease}

Gastric or duodenal ulcer; chronic inflammatory bowel disease

Medical history; current treatment

Medical history of hepatitis including type of hepatitis $(A, B, C, E)$ and liver cirrhosis; current treatment

Hepatitis immunoassay and PCR-based confirmatory test results for hepatitis B and C

Serum transaminases (ALT, AST), cholestatic liver enzymes (AP, GGT), lipase; bilirubinuria, urobilinogenuria

\section{Malignant disease}

Any type of cancer

Medical history including type of malignancy; current treatment and cancer follow-up

\section{Mental health disorders ${ }^{c}$}

Anxiety disorder; depression; burnout;

eating disorder

Medical history, current treatment

\section{Musculoskeletal disease}

Osteoarthritis

Medical history and history of joint replacement; current treatment, specialist care; joint pain; lower back pain

Rheumatoid arthritis 


\section{Table 3 Chronic health conditions and information available from health interview ${ }^{a}$ and examination ${ }^{b}$ in DEGS1} (Continued)

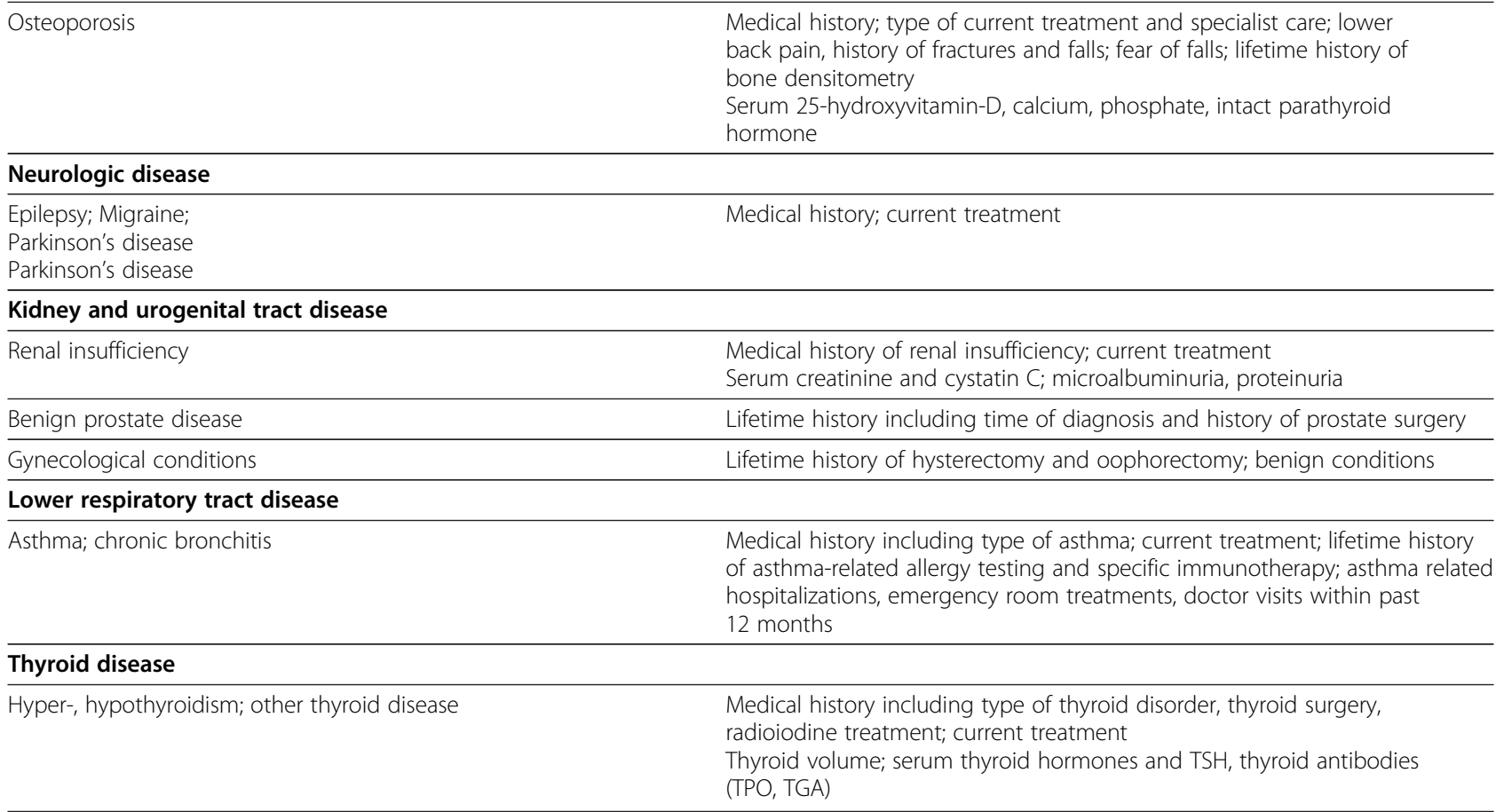

\footnotetext{
${ }^{a}$ Information from computer-assisted personal interviews (CAPI) except for history of benign prostate disease, gynecological conditions, history of falls and fractures, and fear of falls (self-administered questionnaires).

bInformation from standardized measurements and laboratory tests.

Information collected in DEGS1 by CAPI, not related to additional information collected in the DEGS1 mental health study module (DEGS1-MH).

${ }^{d}$ CCP antibody testing was limited to the first year of data collection.

Abbreviations: ALT alanine aminotransferase, AP alkaline phospatase, AST aspartate aminotransferase, GGT gamma-glutamyl transferase, TGA thyreoglobulin antibody, TPO thyroid peroxidase antibody, TSH thyroid stimulating hormone.
}

the Wechsler Adult Intelligence Scale, 3rd revision (WAIS-III).

Health risks and resources covered in DEGS1 include potentially modifiable behavioral risk factors (e.g. dietary habits, tobacco use, and risky alcohol consumption), environmental factors (e.g. living and working conditions, social support, lay caregiving), socio-demographic context variables, and health care services utilization. The FFQ was self-developed and designed to estimate the frequency and amounts of 53 food groups consumed during the past 4 weeks. It was validated against two non-consecutive 24 hour recalls and showed an overall reasonable validity [16]. Compared to the FFQ applied in the GNHIES98, the DEGS1 FFQ has more food items and includes estimation of portion sizes, which permits a more quantitative estimation of food intake. The instruments also cover a different time frame. Alcohol consumption and tobacco use were assessed by selfdeveloped questions already applied in the GNHIES98. Validated German versions of established instruments were additionally applied in DEGS1 to identify persons with risky drinking behavior or nicotine dependence. Socio-demographic variables were assessed according to standards recommended by the German Epidemiology and Preventive Medicine Associations [17]. Education questions meet the International Standard Classification of Education (ISCED) [18].

\section{DEGS1 Mental Health Study Module}

A separate mental health study module (DEGS1-MH) was implemented for in depth assessment of mental health disorders, help-seeking behavior and cognitive function. The data were collected using computerassisted face-to-face interviews administered by specifically trained psychologists. DEGS1 participants were asked for their consent to be recontacted for a study center or at home appointment within four weeks after the basic DEGS1 clinic visit.

The core instrument of DEGS1-MH is a computerassisted German version of the Munich Composite International Diagnostic Interview (DIA-X/M-CIDI) [19], a modified and translated version of the World 
Health Organization Composite International Diagnostic Interview (CIDI) [20]. The CIDI is a fully structured interview for the assessment of mental disorders according to the definitions and criteria of ICD-10 and DSM-IV. It consists of several sections tailored to assess mood disorders (major depression, dysthymic disorder, bipolar disorder); anxiety disorders (panic disorder, specific phobias, agoraphobia, generalized anxiety disorder, social phobia); post-traumatic stress disorder; obsessive-compulsive disorder; substance use disorders (alcohol abuse and dependence, abuse and dependence of pharmaceuticals, nicotine dependence); eating disorders; somatoform disorders (pain disorders, undifferentiated somatoform disorder); psychotic symptoms (screening without further differential diagnosis); treatment of mental disorders. The CIDI is a diagnostic instrument intended for use in clinical practice as well as in clinical and epidemiological research. It is suitable for use in population-based studies to estimate the prevalence of mental disorders, to assess severity of mental disorders and associated burden of disease, and to analyze treatment patterns, treatment barriers, and aspects of quality of care $[20,21]$.

Cognitive function was assessed with a neuropsychological test battery that covers important cognitive domains such as episodic memory, working memory, executive function, and cognitive speed. The battery consists of established cognitive tests that were used in epidemiological studies before and were mostly derived from widely-used test batteries such as the German version of the CERAD battery [22,23].

The DEGS1-MH is carried out in close cooperation between the Robert Koch Institute and the Institute of Clinical Psychology and Psychotherapy, Center of Clinical Epidemiology and Longitudinal Studies (CELOS) at the Technische Universität Dresden. CELOS was already commissioned to carry out the Mental Health Module of the GNHIES98 [24,25]. This opens the perspective for analyses of changes in the prevalence of mental health problems as well as for longitudinal analyses based on individuals participating in both surveys.

\section{Assessment of non-response bias}

Persons sampled and eligible for DEGS1 who declined participation were asked to provide the reason for nonparticipation. In addition, they were asked to fill in a short questionnaire covering essential health-related characteristics, such as self-rated health, history of longstanding chronic illness, smoking habits, educational background, and doctor visits. This will permit comparison of participants and non-participants with respect to age, sex, education, health status, health-related behavior, and health care services utilization.

\section{Data management and preparation for analysis}

The DEGS1 data set will comprise approximately 2000 items per study participant. Data management has been carried out in parallel with data collection based on highly standardized and partly automated procedures for data processing and plausibility checking. This will assist in providing high quality and timely data. A final set of key data was available for main pre-planned analyses 6 months after the completion of fieldwork. Data from the DEGS1 survey wave will be made available to the scientific community as a public use file in 2014 .

In order to assure that estimates derived from DEGS1 are representative at the national level, two sets of sample weights were computed :one set for all participants aged 18-79 years, one set for those with examination data available. Sample weights adjust for different sampling probabilities within the design strata and correct for deviations between the design-weighted net sample and German population statistics (December 31, 2010) based on an iterative raking procedure using age, sex, federal state of residence, community BIK classification category, nationality (German yes/no), and education level according to ISCED [18] using population statistics dating from 2009. For GNHIES98 participants included in DEGS1, the weighting procedure additionally includes adjustments for the re-participation rate.

\section{Quality assurance}

The DEGS1 study operations manual contains standard operating procedures (SOPs) for all DEGS1 interview and examination components as well as internal and external quality control measures [8]. Members of the study teams underwent an initial two-week training session leading to accreditation. They were continuously supervised and reassessed at 6-month intervals. Internal quality control was carried out by senior RKI supervising staff. Measures of internal quality assurance encompassed: regular field visits to the study teams, bimonthly reviews of logbook entries for meeting requirements of daily calibration procedures and transportation of blood and urine specimens, continuous data checks for completeness and plausibility. External quality assurance was carried out by specifically experienced scientists of the Bremen Institute for Epidemiology and Prevention Research (BIPS) based on field observation and data audits.

\section{Feed-back to study participants}

Participants received immediate feed-back on clinically relevant test results based on written summary reports 
prepared by study physicians. Within six to eight weeks, study participants received a standardized letter summarizing clinically relevant study results including clinical chemistry, test results for hepatitis, chlamydia and gonorrhea infections, specific allergy testing, body mass index, blood pressure, thyroid volume, and physical fitness. Study participants will also be informed about immunity status against poliomyelitis, measles, mumps, and rubella as test results become available.

\section{Collaborations}

The DEGS1 steering committee at the Robert Koch Institute welcomes collaborations with external researchers. Collaborations may relate to input of specific knowhow and professional expertise to analyses that were given a particularly high level of priority and therefore pre-planned to be completed within two years after finishing fieldwork. Additional analyses will require a research proposal, which will be reviewed by the DEGS1 steering committee and the Robert Koch Institute Research Council in order to ensure data protection and privacy regulations, public health relevance, data efficiency, and scientific quality. For further information please visit the DEGS1 study homepage at www.degs-studie.de.

\section{Discussion}

As part of the recently established continuous health monitoring system in Germany, the first data collection wave of the German Health Interview and Examination Survey for Adults (DEGS1) was conducted by the Robert Koch Institute between November 2008 and December 2011. The DEGS national health survey system is primarily designed to provide nationally representative health interview and examination data for the population 18-79 years of age and to conduct analyses of time trends in population health and disability. Interview and examination data will be collected in periodically repeated survey waves at 8-year intervals [6]. By including a large number of persons who already participated in a previous national health interview and examination survey (GNHIES98), DEGS1 combines a national health survey and a survey follow-up study. In order to keep cross-sectional results of DEGS1 representative at the national level, a number of measures were taken. First, to compensate for attrition and aging of the GNHIES98 cohort, a sample of the population 18-79 years was newly drawn from local population registries using a two-stage stratified cluster sampling procedure. Secondly, results from DEGS1 cross-sectional analyses will be weighted by survey-specific weights considering sampling probabilities and non-response for newly drawn participants as well as re-participation probability for GNHIES98 participants.
In DEGS1, a wide range of objective health measures were collected based on highly standardized measurements and tests including laboratory analyses. Automated medication assessment and coding provided detailed and objective information on current medication use. It will hence be possible to verify self-reported health conditions, to identify undetected cases of disease, and to evaluate current disease or risk factor control in the population. High risk groups and health inequalities will be identified based on analyses stratified by sex, age, educational background, income, professional and employment status, and region of residence. Burden of disease will be assessed by analyzing the association of morbidity and comorbidity with functional impairment, subjective health, health-related quality of life, and disability. In order to explore preventive potential, these associations will be further analyzed with respect to personal and environmental resources, e.g. socio-demographic characteristics, health-related behavior, level of perceived social support, health care services utilization.

Results of trend analyses will be used to identify new health risks, to evaluate the effectiveness of health programs and regulations at the population level (e.g. reduction of smoking rates; improved hypertension control), and to validate absolute risk prediction models. Interpretation of time trends will need to consider survey data as well as additional information, such as trends in cause-specific mortality, disease management program enrollment, treatment patterns, ambulatory care sensitive hospital admissions, and nursing care rates. As an example, the prevalence of persons with known diabetes mellitus is likely to have increased over time due to aging of the population and increases in the prevalence of major diabetes risk factors such as obesity. However, improved awareness, treatment and survival may have contributed to increases in prevalence as well. Further insight will be gained from comparative analyses of time trends in the prevalence of persons with known and undetected diabetes.

With respect to time trend analyses, concepts, indicators, instruments and methods of data collection in DEGS1 were kept the same as in prior national health interview and examination surveys, in particular the GNHIES98, as far as possible. There were certain limitations to this. For example, there was a change in methods for measuring blood pressure and some biochemical measures. Analyses of time trends will hence require cross-calibration studies in some cases. Furthermore, several constructs and instruments were newly added in DEGS1. Health indicators have been continuously reviewed and extended in close cooperation with health policy makers and public health expert groups, in order to permit harmonization with regional population-based 
studies in Germany and with national health surveys from other countries. In order to improve the comparability of results between countries, great efforts have been undertaken at the EU and OECD level to harmonize indicators and instruments and to rigorously standardize the data collection process [26-29]. Areas of ongoing work on indicators particularly relate to health in older age [30].

DEGS1 response rates among newly recruited study participants are considerably lower than response rates in the GNHIES98 (42\% vs. 62\%). Declining response rates in population-based health surveys have consistently been reported from other European countries over the past decade [31,32]. Selection bias resulting from selective participation of healthier persons is a concern in any population-based survey [33]. Survey results may therefore underestimate the overall prevalence of chronic diseases and disability compared to results from claims data [34]. In addition, persons unable to provide written consent and those with significant language barriers were excluded from participation in DEGS1. Thus, DEGS1 is likely to underrepresent adults living in institutions. To some extent, this may also be true for persons with an immigration background, even though sample weights encompass adjustment for nationality (German yes/no). Careful non-response analyses will be carried out based on available information from official health statistics and non-response questionnaires with respect to nursing care enrollment and institutionalization, non-German nationality, self-rated health, history of long-standing chronic illness, smoking habits, educational background, and health care services utilization.

About half of the DEGS1 study population already participated in a previous national health survey (GNHIES98). This provides ten- to 12-year follow-up data on nearly 4000 individuals. It will hence be possible to analyze age- and sex-specific individual level changes in many risk factors and health status indicators available from both surveys as presented in Table $2[8,16-$ 18,35-66]. The DEGS survey panel will continuously grow and timepoints of measurements will be added as DEGS participants who agreed to be re-contacted will be included in subsequent survey interview and examination waves. In between examination survey waves, DEGS participants will be contacted by telephone and postal questionnaires, in order to assess incident health events and changes in health status and health-related behavior. The panel will be continuously followed for vital status and cause-specific death. Participants will be asked for advance written permission for death certificate review, in order to assess cause-specific mortality. Together, this growing data base will facilitate to study trajectories of risk factors, chronic conditions, and health outcomes. In addition, extended follow-up will increase absolute numbers for estimates of disease incidence and prospective analyses using incident health events and total or cause-specific deaths as outcome measures [6].

In summary, data from DEGS1 and subsequent DEGS survey waves will make essential contributions to the surveillance of communicable as well as noncommunicable diseases in Germany. Data collected in periodically repeated national health surveys of adults in Germany will be used for continuous health reporting. Findings will be relevant to health policy planning and evaluation, public health research, and health information of the lay public. Survey participants will be followed for changes in health status and health outcomes. Cross-sectional and longitudinal data will be entered in a large health data platform for health and health care services research.

\section{Competing interests}

The authors declare that they have no competing interests.

\section{Authors' contributions}

BMK, PK, CSN conceptualized and supervised the study and wrote the final manuscript. $\mathrm{AG}, \mathrm{ML}$ and $\mathrm{HH}$ oversaw the field work and quality assurance of data, assisted in conceptualizing the study and contributed to writing the manuscript. MB, SD, RD, UE, JF, UH, CH, HK, DL, GBM, HN, AR, AS, ASR, HS, MT provided specific knowledge, contributed to the conceptualization of the study and the quality assurance of data, as well as to the writing of the final manuscript. All authors read and approved the final manuscript.

\section{Acknowledgements}

DEGS1 is primarily funded by the German Ministry of Health (BMG). Supplementary funding of DEGS1 is provided by the German Ministry of Food, Agriculture, and Consumer Protection (BMELV) for monitoring of allergic sensitization, and by the German Association of Psychotherapy and Psychiatry (DGPPN) for implementing the module on mental health. This study could not have been realized without the support of so many more colleagues in the Robert Koch Institute. We specifically thank the study teams for their excellent work and great commitment during three years of data collection. We are indebted to the mayors and authorities of the 180 sampled communities for supporting the field logistics. We would like to acknowledge Daniel Grams and Lina Nowka for providing assistance in preparing the manuscript. Above all, we wish to thank all the DEGS1 study participants.

Received: 24 August 2012 Accepted: 28 August 2012

Published: 1 September 2012

\section{References}

1. Hoffmeister $H$, Mensink GB, Stolzenberg H: National trends in risk factors for cardiovascular disease in Germany. Prev Med 1994, 23:197-205.

2. Bellach BM, Knopf H, Thefeld W: The German Health Survey 1997/98 (article in German). Gesundheitswesen 1998, 60(Suppl 2):59-68.

3. Hermann-Kunz E: Prevalence of hay fever in Germany - comparison between Eastern and Western Germany and time trends (in German) Gesundheitswesen 1999, 61(Spec No):94-99.

4. Kurth BM, Kamtsiuris $P$, Hölling H, Schlaud M, Dölle R, Ellert U, Kahl H, Knopf $H$, Lange M, Mensink GB, Neuhauser H, Rosario AS, Scheidt-Nave C, Schenk L, Schlack R, Stolzenberg H, Thamm M, Thierfelder W, Wolf U: The challenge of comprehensively mapping children's health in a nation-wide health survey: design of the German KiGGS-Study. BMC Publ Health 2008, 8:196

5. Kurth BM, Lange C, Kamtsiuris $\mathrm{P}$, Hölling $\mathrm{H}$ : Health monitoring at the Robert Koch Institute. Status and perspectives (in German). 
Bundesgesundheitsblatt Gesundheitsforschung Gesundheitsschutz 2009, 52:557-570.

6. Gößwald A, Lange M, Kamtsiuris P, Kurth BM: DEGS: German Health Interview and Examination Survey for Adults: A nationwide crosssectional and longitudinal study within the framework of health monitoring conducted by the Robert Koch Institute (in German). Bundesgesundheitsblatt Gesundheitsforschung Gesundheitsschutz 2012, 55:775-780.

7. The World Medical Association (WMA): Declaration of Helsinki - Ethical principles of medical research involving human subjects. http://www.wma.net/en/30publications/10policies/b3/.

8. Robert Koch-Institut: DEGS-Studie zur Gesundheit Erwachsener in Deutschland. Projektbeschreibung. Berlin: Robert Koch-Institut; 2009.

9. BIK Aschpurwis und Behrens GmbH: BIK Regionen: Ballungsräume, Stadtregionen, Mittel-/Unterzentrengebiete. Methodenbeschreibung zur Aktualisierung 2000. Hamburg; 2001.

10. Cox LH: A constructive procedure for unbiased controlled rounding. J Am Stat Assoc 1987, 82:520-524.

11. Kalton G: Introduction to Survey Sampling. Beverly Hills: Sage Publications; 1983

12. Kish L: Survey Sampling. New York: John Wiley \& Sons; 1965.

13. Newcombe RG: Two-sided confidence intervals for the single proportion: comparison of seven methods. Stat Med 1998, 17:857-872.

14. Collaborating Centre for Drug Statistics Methodology: Guidelines for ATC classification and DDD assignment 2010. http://www.whocc.no/filearchive/publications/2010guidelines.pdf.

15. American College of Sports Medicine: ACSM's Guidelines for exercise testing and prescription 8th edition. Philadelphia: Lippincott Williams \& Wilkins; 2010.

16. Haftenberger M, Heuer T, Heidemann C, Kube F, Krems C, Mensink GB: Relative validation of a food frequency questionnaire for national health and nutrition monitoring. Nutr J 2010, 9:36.

17. Jöckel KH, Babitsch B, Bellach BM, Bloomfield K, Hoffmeyer-Zlotnik J, Winkler J: Empfehlungen der Arbeitsgruppe "Epidemiologische Methoden" in der Deutschen Arbeitsgemeinschaft Epidemiologie der Gesellschaft für Medizinische Informatik, Biometrie und Epidemiologie (GMDS) und der Deutschen Gesellschaft für Sozialmedizin und Prävention (DGSMP) zur Messung und Quantifizierung soziodemographischer Merkmale in epidemiologischen Studien. In Messung soziodemographischer Merkmale in der Epidemiologie. Volume 1. Edited by Ahrens W, Bellach BM, Jöckel KH. München: MMV Medizin Verlag; 1998.

18. Schroedter JH, Lechert Y, Lüttiger P: Die Umsetzung der Bildungsskala ISCED-1997 für die Volkszählung 1970, die Mikrozensus-Zusatzerhebung 1971 und die Mikrozensen 1976-2004 (Version 1). In ZUMAMethodenbericht 2006/08. Edited by Zentrum für Umfragen, Methoden und Analysen - ZUMA. Mannheim: 2006

19. Wittchen HU, Pfister H: DIA-X-Interviews: Manual für Screening-Verfahren und Interview. Frankfurt: Swets \& Zeitlinger; 1997

20. Kessler RC, Ustün TB: The World Mental Health (WMH) Survey Initiative Version of the World Health Organization (WHO) Composite International Diagnostic Interview (CIDI). Int J Methods Psychiatr Res 2004 13:93-121.

21. Wittchen HU, Kessler RC, Ustün TB: Properties of the Composite International Diagnostic Interview (CIDI) for measuring mental health outcome. In Mental Health Outcome Measures. 2nd edition. Edited by Thornicroft G, Tansella M. London: Gaskell; 2001:212-227.

22. Morris JC, Heyman A, Mohs RC, Hughes JP, van Belle G, Fillenbaum G, Mellits ED, Clark C: The Consortium to Establish a Registry for Alzheimer's Disease (CERAD). Part I. Clinical and neuropsychological assessment of Alzheimer's disease. Neurology 1989, 39:1159-1165.

23. Luck T, Riedel-Heller SG, Wiese B, Stein J, Weyerer S, Werle J, Kaduszkiewicz $H$, Wagner M, Mösch E, Zimmermann T, Maier W, Bickel H, van den Bussche $H$, Jessen F, Fuchs A, Pentzek M, AgeCoDe Study Group: CERAD-NP battery: Age-, gender- and education-specific reference values for selected subtests. Results of the German Study on Ageing, Cognition and Dementia in Primary Care Patients (AgeCoDe). Z Gerontol Geriatr 2009, 42:372-384.

24. Jacobi F, Wittchen HU, Hölting C, Sommer S, Lieb R, Höfler M, Pfister H: Estimating the prevalence of mental and somatic disorders in the community: aims and methods of the German National Health Interview and Examination Survey. Int J Methods Psychiatr Res 2002, 11:1-18.
25. Jacobi F, Wittchen HU, Holting C, Höfler M, Pfister H, Müller N, Lieb R: Prevalence, co-morbidity and correlates of mental disorders in the general population: results from the German Health Interview and Examination Survey (GHS). Psychol Med 2004, 34:597-611.

26. Tolonen H, Koponen P, Aromaa A, Conti S, Graff-Iversen S, Grøtvedt L, Kanieff M, Minell J, Natunen S, Primatesta P, Verschuren Viet L: Kuulasmaa K for the Feasibility of a European Health Examination Survey (FEHES) Project: Review of Health Examination Sunveys in Europe. http://www.ktl.fi/attachments/suomi/julkaisut/julkaisusarja_b/2008/2008b18.pdf.

27. Tafforeau J, Cobo ML, Tolonen H, Scheidt-Nave C, Tinto A: Guidelines for the development and criteria for the adoption of Health Survey instruments. http:// circa.europa.eu/Public/irc/dsis/health/library?l=/reports/

healthsinterviewssurvey/guidelines_instruments/_EN_1.0_\&a=d.

28. OECD: Health at a Glance 2011: OECD indicators. http://www.oecd.org/dataoecd/6/28/49105858.pdf.

29. National Institute for Health and Welfare: European Health Examination Survey. http://www.ehes.info.

30. Holzhausen M, Fuchs J, Busch M, Ernert A, Six-Merker J, Knopf H, Hapke U, Gaertner B, Kurzawe-Seitz I, Dietzel R, Schödel N, Welke J, Wiskott J, Wetzstein M, Martus P, Scheidt-Nave C: Operationalizing multimorbidity and autonomy for health services research in aging populations-the OMAHA study. BMC Health Serv Res 2011, 11:47.

31. De Heer W: International Response Trends: Results of an International Survey. J Off Stat 1999, 15:129-142.

32. Tolonen H, Helakorpi S, Talala K, Helasoja V, Martelin T, Prättälä R: 25-year trends and socio-demographic differences in response rates: Finnish adult health behaviour survey. Eur J Epidemiol 2006, 21:409-415.

33. Criqui $M$, Barrett-Connor $E$, Austin M: Differences between respondents and non-respondents in a population-based cardiovascular disease study. Am J Epidemiol 1978, 108:367-372.

34. Naughton C, Bennett K, Feely J: Prevalence of chronic disease in the elderly based on a national pharmacy claims database. Age Ageing 2006, 35:633-636

35. Cox B, Van Oyen H, Cambois E, Jagger C, le Roy S, Robine JM, Romieu I: The reliability of the Minimum European Health Module. Int $\mathrm{J}$ Public Health 2009, 54:55-60.

36. Franke GH: SCL-90-R. Die Symptom-Checkliste von Derogatis. Deutsche Version - Manual. Zweite, vollständig überarbeitete und neu normierte Auflage. Göttingen: Beltz; 2002.

37. Fransen J, Langenegger T, Michel BA, Stucki G: Feasibility and validity of the RADAl, a self-administered rheumatoid arthritis disease activity index. Rheumatology (Oxford) 2000, 39:321-327.

38. Ware JE, Kosinski M, Bjorner JB, Turner-Bowker DM, Gandek B, Marusih ME: User's manual for the SF-36V2 health survey. 2nd edition. Lincoln, RI: Quality Metric Incorporated; 2007.

39. Morfeld M, Bullinger M, Nantke J, Brähler E: The version 2.0 of the SF-36 Health Survey: results of a population-representative study (in German). Soz Praventivmed 2005, 50:292-300.

40. Kurth BM, Ellert U: The SF-36 questionnaire and its usefulness in population studies: results of the German Health Interview and Examination Survey 1998. Soz Praventivmed 2002, 47:266-277.

41. Riemann D, Backhaus J: Behandlung von Schlafstörungen. Weinheim: Psychologie Verlags Union; 1996.

42. Kroenke K, Spitzer RL, Williams JB: The PHQ-9: validity of a brief depression severity measure. J Gen Intern Med 2001, 16:606-613.

43. Löwe B, Spitzer RL, Zipfel S, Herzog W: PRIME MD Patient Health Questionnaire (PHQ). 2nd edition. http://www.klinikum.uniheidelberg.de/ fileadmin/Psychosomatische_Klinik/download/PHQ_Manual1.pdf.

44. Schulz P, Schlotz W: The Trier Inventory for the Assessment of Chronic Stress (TICS): Scale construction, statistical testing, and validation of the scale work overload. Diagnostica 1999, 45:8-19.

45. Schulz P, Schlotz W, Becker P: Das Trierer Inventar zum chronischen Stress (TICS) - Manual. Göttingen: Hogrefe; 2004.

46. Rumpf HJ, Meyer C, Hapke U, John U: Screening for mental health: Validity of MHI-5 using DSM-IV Axis I psychiatric disorders as gold standard Psychiatry Res 2001, 105:243-253.

47. DZA (German Centre of Gerontology): German Aging Survey (DEAS) 2002 Interview. http://www.dza.de/fileadmin/dza/pdf/English/DEAS2002_Interview. pdf.

48. Ismail AA, O'Neill TW, Cockerill W, Finn JD, Cannata JB, Hoszowski K, Johnell O, Matthis C, Raspe H, Raspe A, Reeve J, Silman AJ: Validity of self-report of 
fractures: results from a prospective study in men and women across Europe. EPOS Study Group. European Prospective Osteoporosis Study Group. Osteoporos Int 2000, 11:248-254.

49. Lamb SE, Jorstad-Stein EC, Hauer K, Becker C: Development of a common outcome data set for fall injury prevention trials: the Prevention of Falls Network Europe consensus. J Am Geriatr Soc 2005, 53:1618-1622

50. Kilpeläinen K, Aromaa A, ECHIM Core Group: European Health Indicators: Development and Initial Implementation.Final Report of the European Health Indicator Monitoring (ECHIM) Projekt. http://www.echim.org/docs/ ECHIM_final_report.pdf.

51. Gore CJ, Booth ML, Bauman A, Owen N: Utility of pwc $75 \%$ as an estimate of aerobic power in epidemiological and population-based studies. Med Sci Sports Exerc 1999, 31:348-351.

52. Lange Andersen K, Shephard RJ, Denolin H, Varnauskas E, Masironi R: Fundamentals of Exercise Testing. Geneva: World Health Organisation; 1971.

53. Mohd Hairi F, Mackenbach JP, Andersen-Ranberg K, Avendano M: Does socio-economic status predict grip strength in older Europeans? Results from the SHARE study in non-institutionalised men and women aged 50+. J Epidemiol Community Health 2010, 64:829-837.

54. Hank K, Jürges H, Schupp J, Wagner GG: Isometric grip strength and social gerontological research: results and analytic potentials of SHARE and SOEP (in German). Z Gerontol Geriatr 2009, 42:117-126.

55. Podsiadlo D, Richardson S: The timed "Up \& Go": a test of basic functional mobility for frail elderly persons. J Am Geriatr Soc 1991, 39:142-148.

56. Guralnik JM, Simonsick EM, Ferrucci L, Glynn R, Berkman LF, Blazer DG, Scherr PA, Wallace RB: A short physical performance battery assessing lower extremity function: association with self-reported disability and prediction of mortality and nursing home admission. J Gerontol 1994, 49:M85-M94.

57. Guralnik JM, Ferrucci L, Simonsick EM, Salive ME, Wallace RB: Lower extremity function in persons over the age of 70 years as a predictor of subsequent disability. New Engl J Med 1995, 332:556-561.

58. Rossiter-Fornoff JE, Wolf SL, Wolfson LI, Buchner DM: A cross-sectional validation study of the FICSIT common data base static balance measures. Frailty and Injuries: Cooperative Studies of Intervention Techniques. J Gerontol A Biol Sci Med Sci 1995, 50:M291-M297.

59. von Aster M, Neubauer A, Horn R: Wechsler Intelligenztest für Erwachsene. Frankfurt/M: Harcourt Test Services; 2006.

60. Wechsler D: Wechsler Adult Intelligence Scale - third edition. San Antonio, TX: The Psychological Corporation; 1997.

61. Jagger C, Gillies C, Cambois E, Van Oyen H, Nusselder W, Robine J-M, the EHLEIS team: The Global Activity Limitation Indicator (GALI) measured function and disability similarly across European countries. J Clin Epidemiol 2010, 63:892-899.

62. John U, Meyer C, Hapke U, Rumpf HJ, Schumann A, Adam C, Alte D, Lüdemann J: The Fagerström Test for Nicotine Dependence in two adult population samples. Alcohol Drug Depend 2003, 20:1-6.

63. Bischof G, Reinhardt S, Grothues J, Meyer C, John U, Rumpf HJ: Development and evaluation of a screening instrument for alcohol-use disorders and at-risk drinking: the brief alcohol screening instrument for medical care (BASIC). J Stud Alcohol Drugs 2007, 68:607-614.

64. Dalgard OS, Dowrick C, Lehtinen V, Vazquez-Barquero JL, Casey P, Wilkinson G, Ayuso-Mateos JL, Page H, Dunn G, ODIN Group: Negative life events, social support and gender difference in depression: a multinational community survey with data from the ODIN study. Soc Psychiatry Psychiatr Epidemiol 2006, 41:444-451.

65. Schenk L, Bau AM, Borde T, Butler J, Lampert T, Neuhauser H, Weilandt C: A basic set of indicators for mapping migrant status. Recommendations for epidemiological practice (in German). Bundesgesundheitsblatt Gesundheitsforschung Gesundheitsschutz 2006, 49:853-860.

66. Thamm M, Ellert $U$, Thierfelder W, Liesenkötter KP, Völzke H: lodine intake in Germany. Results of iodine monitoring in the German Health Interview and Examination Survey for Children and Adolescents (KiGGS) (in German). Bundesgesundheitsblatt Gesundheitsforschung Gesundheitsschutz 2007, 50:744-749.

doi:10.1186/1471-2458-12-730

Cite this article as: Scheidt-Nave et al:: German health interview and examination survey for adults (DEGS) - design, objectives and implementation of the first data collection wave. BMC Public Health 2012 $12: 730$

\section{Submit your next manuscript to BioMed Central and take full advantage of:}

- Convenient online submission

- Thorough peer review

- No space constraints or color figure charges

- Immediate publication on acceptance

- Inclusion in PubMed, CAS, Scopus and Google Scholar

- Research which is freely available for redistribution 